\title{
Design of 2-DOF PID Compensator for Magnetic Levitation System
}

\author{
Navaneethan $\mathrm{S}^{1}$, Olivia Merlyn $\mathrm{J}^{2}$ \\ \{snn.ice@psgtech.ac.in ${ }^{1}$, jmerlyn3139@gmail.com² ${ }^{2}$,
}

Assistant Professor, Department of Instrumentation and Control Systems Engineering

PSG College of Technology, Coimbatore,Tamilnadu ${ }^{1}$, PG scholar, Department of Instrumentation and Control Systems Engineering, PSG College of Technology, Coimbatore,Tamilnadu. ${ }^{2}$

\begin{abstract}
In recent years there are many advancements in the field of electromagnetics one among them is the development of Magnetic Levitation (Maglev) system. It has found various uses in fields such as high speed magnetic levitation trains, frictionless bearings, wind tunnel model levitation, vibration isolation of sensitive machinery, molten metal levitation in induction furnaces., lifts, conveyors etc. When this technology is applied to practical systems it will be affected by external disturbance and set point changes at the same time. So a multi-lens driver is required. Furthermore, the opencircuit maglev system is a highly non-linear and unstable system. Developing a proper control for such a system is really a demanding task.In this paper a 2-DOF PID compensator for the Maglev system is designed. Simulation is done using MATLAB and the results are compared with 1-DOF method. The additional feed forward components present improves the disturbance rejection capability and makes the system more stable when compared to 1-DOF controller. It is proved that 2-DOF controller provides both tracking and regulatory performance in an optimal manner. .
\end{abstract}

Keywords: Magnetic Levitation, 2-DOF PID Compensation.

\section{Introduction}

Magnetic levitation technology is a non-contact technology. This technology reduces physical contact between moving and fixed parts and, in turn, eliminates the problem of friction. Allows hanging objects almost without problems. Only the magnetic force generated by the electromagnets of the magnetic levitation system transports the levitated object. In addition to low friction, other advantages are the ability to actively change the position of the suspended object and change the properties (eg stiffness) of the suspension system. Due to their advantages, magnetic levitation systems are used in various fields of application such as traction, electrical appliances, wind turbines, etc.

Magnetic Levitation system is highly nonlinear and unstable system. So many researchers have tried many different controllers for this system. Some of them are listed below. As Maglev system is nonlinear system many nonlinear techniques are also applied for it. Rafael Morales et.al.,treatsmagnetic levitation systems from the perspective of adaptive control based on rapid estimation of online algebraic parameters, exact linearization and generalized proportional-integral (GPI) output feedback control. This controller was able to get better results for both stabilization and performance tracking. This is the first work that has 
successfully applied the algebraic identification method to a highly non-linear, unstable and insecure experimental system. [3] MeiYung Chen et al. features self-adjusting adaptive control (STA control) for a magnetic levitation guidance system. In this, a stabilizing STA controller is developed and the comprehensive analysis of the stability property is discussed [5].ZiJiang Yang et.al proposed a powerful adaptive non-linear controller for the position tracking problem of magnetic levitation systems. The combination of adaptive and robust approaches helps to overcome some well-known practical problems such as high gain feedback from robust controllers and poor transient performance of adaptive controllers, so that better control performance can be achieved than if the two were used alone [6].

Neural networks and fuzzy logic controllers have found solutions for many practical systems. Research has tried it also FaaJeng Lin et.al presented the idea of an intelligent scroll mode control system using a radial basis function network (SMCRBFN). RGBFN has the property of faster convergence than multilayer perceptron (MLP) because only the connecting weight between the hidden layer and the network output layer is adjusted during training to reduce computational requirements. This control structure provides the levitation object position of the magnetic levitation system which has the advantages of good transient control performance and uncertainty resistance for tracking periodic trajectories [4]. Tania Tariq Salim et.al presented the differences between the performance of fuzzy logic control (FLC) and LQRC for the same linear model of a magnetic levitation system. Magnetic levitation is a non-linear unstable system and the fuzzy logic controller brings the magnetic levitation system into a stable region by keeping the magnetic ball suspended in the air.

The system must be described with a state space model, so that this type of controller is directly related to the inverse FL controller linear model of the system [7]. RongJong Wai et al dealt with the Adaptive FuzzyNeuralNetwork Control (AFNNC) scheme by imitating the SMC strategy for a maglev transport system. Chattering is a major drawback in the case of sliding mode controllers, so adaptive sliding mode control (ASMC) is being developed [8].

Although many complex control schemes have been tried, advances in PID control have also played an important role in controller design for maglev systems. VinodhKumar.E et.al deals with a 1DOF (Degree Of Freedom) PID control approach in which the gain of the PID controller is determined through a linear quadratic regulator approach. This paper also describes a detailed linearization modeling of the magnetic levitation system. The system modeling is achieved by using Kirchhoff's basic laws of motion and stress laws. Since the Maglev system is a non-linear system, it is linearized around the equilibrium point by applying the Taylor series [2]. MrunalDeshpande presents the PID algorithm with PSO for the Maglev system. PID with PSO is easy to implement, has stable convergence and good computational efficiency. It is observed that PID control laws are easy to implement but have poor adaptability and difficult parameter setting. In this work, the Particle Swarm Optimization (PSO) technique is used to determine this advantage. [10].

ArunGhoshet.al proposed 2DOF PID compensation for magnetic levitation systems. The 2DOF PID control is a multi-lens control. It is capable of optimally providing set point tracking and simultaneous interference rejection. In this document the gain of the PID controller is designed using the pole positioning technique [1].

This document describes the 2DOF PID compensator for the Maglev system. This controller acts as the main lag compensator for the system. The project organization is as follows section 2 deals with the principles and modeling of the Maglev system. Section 3 discusses the 1DOF PID controller, section 4 discusses the 2DOF PID compensation, and section 5 discusses the conclusion and extension of future work. 


\section{Modeling}

The magnetic levitation system is an electromechanical system. It works on the basic principle of magnetization (ie) how poles repel and opposite poles attract each other. The purpose of this system is to lift objects using a magnetic force. Here a magnetic levitation system is used to levitate a ball through the air using an electromagnet. An appropriate current signal must be given to the system to lift the ball to the desired position. The photo sensor is used to determine the position of the ball. A simple block diagram of a magnetic levitation system is shown in Figure 1.

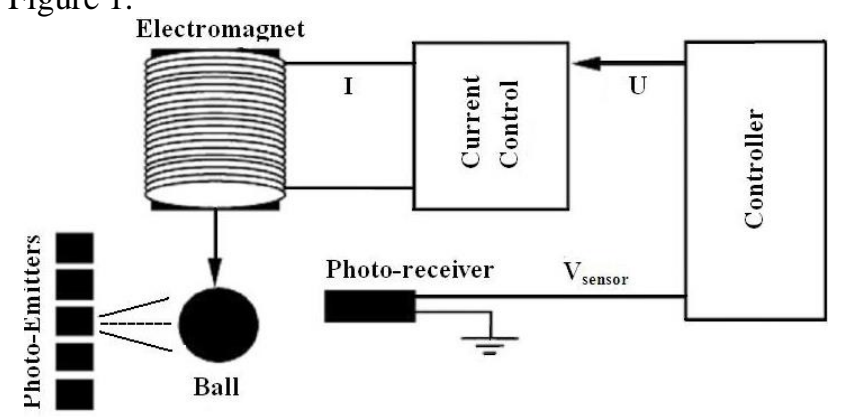

Fig.1.Block diagram of Maglev system

Magnetic levitation system is a nonlinear system and hence deriving a model is challenging task so nonlinear model is derived and it is linearized using Taylor's series. It can be visualized as a simple electro mechanical model. The equivalent circuit diagram of simple maglev system is represented in the Fig 2. This type of modeling is presented in [1],[2]. The whole system can be divided into the electrical and electromechanical system.

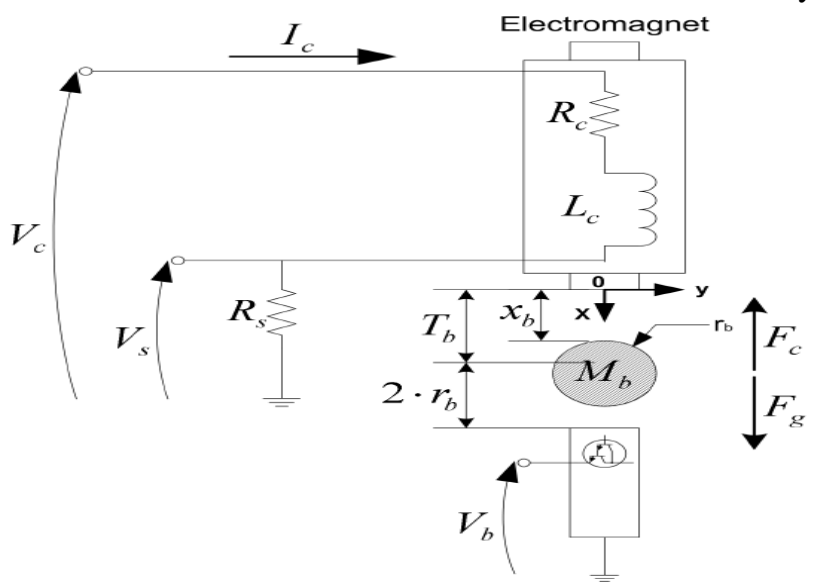

Fig.2. Equivalent circuit of Maglev system

a. Electrical system modeling:

Using Kirchhoff's voltage law, we get the following first-order differential equation:

$\operatorname{Vc}(\mathrm{t})=(\operatorname{Rc}+\operatorname{Rs}) \operatorname{Ic}(\mathrm{t})+\operatorname{Lc}\left(\frac{\mathrm{d}}{\mathrm{dt}} \operatorname{Ic}(\mathrm{t})\right)$

Applying the Laplace transform to above equation and then the desired open-loop transfer function for the Maglev electrical system is 
$\mathrm{Gc}(\mathrm{s})=\frac{\mathrm{Ic}(\mathrm{s})}{\mathrm{Vc}(\mathrm{s})}$

Then the transfer function $\mathrm{Gc}(\mathrm{s})$ is

$\mathrm{Gc}(\mathrm{s})=\frac{\mathrm{Kcdc}}{\tau \mathrm{cs}+1}$

Where,

$$
\begin{gathered}
\mathrm{Kcdc}=\frac{1}{\mathrm{Rc}+\mathrm{RS}} \\
\tau \mathrm{c}=\frac{\mathrm{Lc}}{\mathrm{Rc}+\mathrm{Rs}}
\end{gathered}
$$

The above system is stable because the pole is located on the left-hand side of the splane.

b. Electro-Mechanical System Modelling- Equation of motion:

The force due to gravity applied on the ball is expressed by,

$\mathrm{F}_{\mathrm{g}}=\mathrm{Mg}_{\mathrm{g}}$ (4)

The total external force experienced by the ball using the electromagnet is

$\mathrm{Fc}+\mathrm{Fg}=\frac{-1 * \mathrm{Km} * \mathrm{Ic}{ }^{2}}{\mathrm{Xb}^{2}}+\mathrm{Mb} * \mathrm{~g}$

By using Newton's second law the nonlinear equation of motion is

$\frac{\partial^{2}}{\partial \mathrm{t}^{2}} \mathrm{Xb}=\frac{-1 * \mathrm{Km} * \mathrm{Ic}^{2}}{\mathrm{Mb} * \mathrm{Xb}^{2}}+\mathrm{g}(6)$

At equilibrium the derivative terms equal to zero then,

$\frac{-1 * \mathrm{Km} * \mathrm{Ico}^{2}}{2 * \mathrm{Xbo}^{2}}+\mathrm{Mb} * \mathrm{~g}=0(7)$

The coil current at equilibrium is

Ico $=\sqrt{\frac{2 * \mathrm{Mb} * \mathrm{~g}}{\mathrm{Km}}} \mathrm{Xbo}(8)$

c. Electromechanical system modeling:

The Taylor's series approximation about (Xbo, Ico) is

$\frac{\partial^{2}}{\partial \mathrm{t}^{2}} \mathrm{Xb}=\frac{-1 * \mathrm{Km} * \mathrm{Ic}^{2}}{\mathrm{Mb} * \mathrm{Xb}^{2}}+\mathrm{g}+\frac{\mathrm{Km} * \mathrm{Ico}{ }^{2} * \mathrm{Xbl}}{\mathrm{Mb} * \mathrm{Xbo}^{3}}-\frac{\mathrm{Km} * \mathrm{Ico} * \mathrm{Icl}}{\mathrm{Mb} * \mathrm{Xbo}^{\wedge} 2}(9)$

The desired open-loop current position transfer function of the Maglev electromechanical system after the application of the Laplace transform is

$\operatorname{Gbl}(\mathrm{s})=\frac{\mathrm{Xbl}(\mathrm{s})}{\mathrm{Icl}(\mathrm{s})}$

$\operatorname{Gbl}(s)=\frac{-\mathrm{Kbdc} * \mathrm{wb}^{2}}{\mathrm{~s}^{2}-\mathrm{wb}^{2}}(11)$

Where,

$\mathrm{Kbdc}=\frac{\mathrm{Xbo}}{\mathrm{Ico}}$

$\mathrm{wb}=\sqrt{\frac{2 * g}{\mathrm{Xbo}}}$

The (11) is the overall transfer function of the Maglev system. It is unstable system as one of its pole lie on the right half of the s-plane.

Where,

$I c o=\sqrt{\frac{2 \mathrm{gMb}}{\mathrm{Km}}} * \mathrm{Xbo}$

The basic control objectives of the maglev system are to make the object levitate at a desired position. It consists of two loops the outer loop is used to control desired while the 
inner loop is used to provide proper current signal to the system. The desired position of the ball is achieved by providing proper current signal to the system. So positioning of the ball can be achieved by controlling the voltage applied to the electromagnetic coil. Many controllers are designed to achieve proper positioning of the ball.

\section{1-Dof Pid Controller}

Although many advanced control techniques are used in recent years PID controller also proves to give better performance. Many advancements are applied along with PID controller. The conventional PID controllers used widely are 1-DOF in nature. Degree of freedom deals with the number of controller transfer functions used. 1-DOF controller uses only one controller transfer function. This structure provides either set point tracking or disturbance rejection in optimal manner. 1-DOF controller can satisfy only single objective controller.

The block diagram of a 1-DOF PID controller is as shown Fig 3

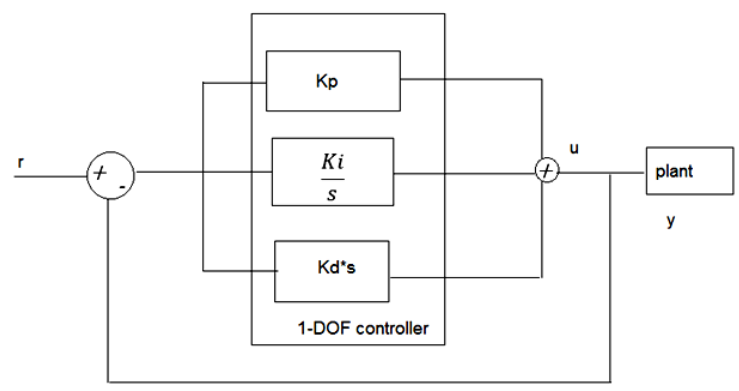

Fig.3 Block diagram of 1-DOF PID controller

The controller transfer function is

$$
u(t)=K p\left(e(t)+\frac{1}{T i} \int e(t) d t+T d \frac{d e(t)}{d t}\right)
$$

In a conventional PID controller the significance of P-term is to improve the transient behavior of the system, the significance of $\mathrm{D}$ - term is to provide better set point tracking. It plays a major role in systems that changes the set point very often, the significance of I-term is to improve the disturbance rejection capability and also to improve the steady state performance of the system. The controller used can be a conventional PID controller. The selection of controller gains or proper tuning of controller enhances the performance of the controller. The gains of the PID controller can be designed using pole placement technique or using Ziegler Nichols training algorithm, Cohan Coon training method

Even though 1-DOF PID controller proves to be better choice there some limitations in the case of disturbance rejection property. It can be easily viewed using the simulation results. The simulation result for 1-DOF PID controller is as shown below. 


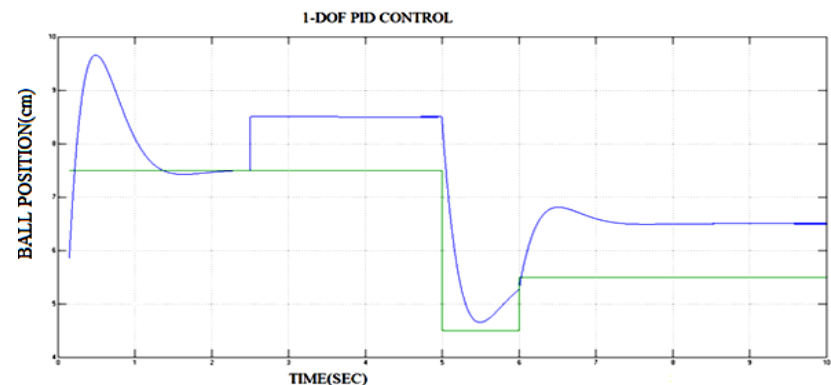

Fig.4 1-DOF PID controller

From the above result it can be inferred that 1-DOF PID controller gives better performance for set point tracking but 1-DOF controller cannot gain back the performance when a disturbance is applied to it. This is because the for disturbance rejection an additional integral component is required which is absent in the case of 1-DOF controller.

As mentioned above Maglev system is an unstable system so the controller designed must stabilize the system. So the next step is to check the relative stability of 1-DOF PID controller. This is done by inserting a gain block before the system. The gain is changed from a nominal value of unity till the system response becomes unstable. The result is as shown in Fig 5

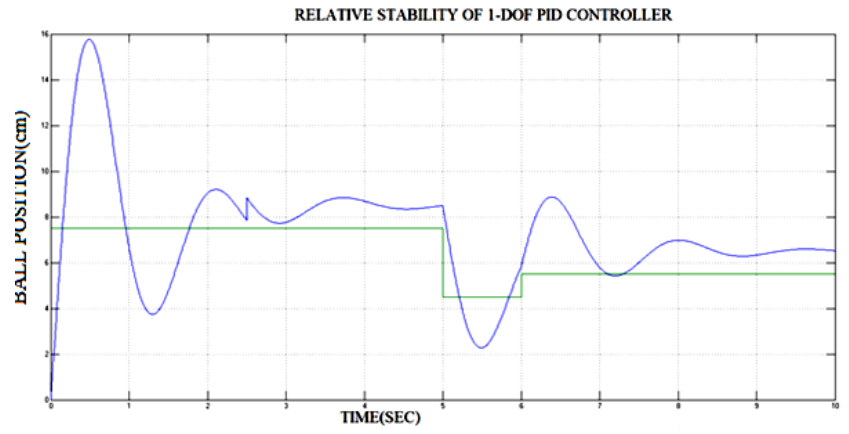

Fig.5 Relative stability of 1-DOF controller

The above result is shown for value of $\mathrm{k}=0.25$. As there are many oscillations in the response the system becomes unstable at this point. This is the relative stability achieved using 1-DOF PID controller.

From the above results it can be inferred that 1-DOF PID controller is not sufficient for disturbance rejection of Maglev system. So a better controller is required to control the system. So the idea of 2-DOF PID compensation is required.

\section{2-Dof Pid Compensation}

2-DOF PID controllers are multi objective controllers (i.e.) same controller can satisfy many control objectives. This decreases the complexity in design of controllers. 2-DOF controllers can satisfy two control objectives. It can provide both set point tracking and disturbance rejection at the same time. These controllers are now in development stage and many new advances are carried out in their design.In many cases an additional feed forward component is present in 2-DOF controllers. This makes the controller a multi objective one. 
The advantages of 2-DOF PID controller is that,

$>$ Both set point tracking and disturbance rejection can be obtained simultaneously in an optimal manner

$>$ The feed forward component clears mostly all the practical considerations of PID controllersand makes it useful for many industrial processes in real time

$>$ It makes the system more stable thus it can be used to stabilize unstable systems.

From the above advantages, it can be inferred that if 2DOF PID control is applied for the Maglev system, it can address both setpoint tracking and interference suppression and also stabilize the system with little control effort. For example, a 2DOF PID compensator has been developed for the Maglev system. The functional diagram of the 2DOF PID controller for the Maglev system is shown in Figure 6

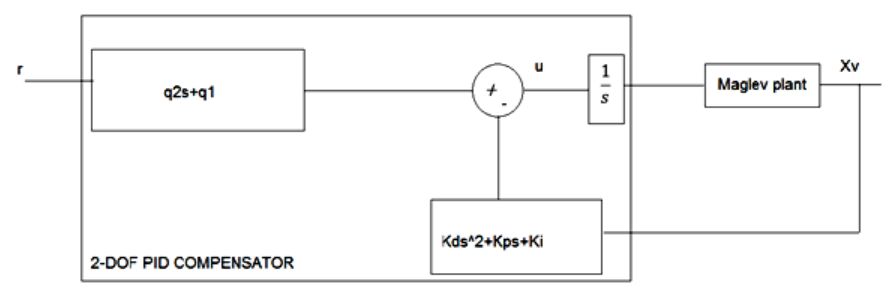

Fig.6 Block diagram of 2-DOF PID compensator for Maglev system

The PID gains are designed using pole placement technique. The additional components $\mathrm{q} 2$ and $\mathrm{q} 1$ are called as feed forward components. The controller transfer function for 2-DOF PID controller is

$\frac{\Delta \mathrm{x}}{\Delta \mathrm{r}}=\frac{(\mathrm{q} 2 \mathrm{~s}+\mathrm{q} 1) \mathrm{b}}{\mathrm{s}^{3}+K d \mathrm{~s}^{2}+\left(\mathrm{bKp}-\mathrm{p}^{2}\right) \mathrm{s}+\mathrm{Kib}}$

There are 5 main steps involved in designing the controller parameters they are as listed below,

1. Set $\mathrm{q} 2=0$.

2. Choose suitable $\xi$ and $\omega$ n corresponding to $\mathrm{q} 2=0$

3. Choose the desired pole $s=-\alpha$ such that it is far away from $s=-\zeta W n$

4. Determine $\mathrm{Kp}, \mathrm{Ki}, \mathrm{Kd}$, and set $\mathrm{q} 1=\mathrm{Ki}$

5. Tune $\mathrm{q} 2$ to obtain the desired speed of the response.

By following the above steps for designing a 2-DOF PID controller the PID gains are,

$\mathrm{Kp}=\frac{\omega \mathrm{n}^{2}+2 \zeta \omega \mathrm{n} \alpha+\mathrm{p}^{2}}{\mathrm{~b}}$

$\mathrm{Kd}=\frac{2 \zeta \omega \mathrm{n}+\alpha}{\mathrm{b}}$

$\mathrm{Ki}=\frac{\omega \mathrm{n}^{2} * \alpha}{\mathrm{b}}$

$\mathrm{q} 1=\mathrm{Ki}$

where,

$\mathrm{b}=\frac{2 \mathrm{~g}}{\mathrm{I} 0 \mathrm{~g}}$

$\mathrm{p}=\frac{2 \mathrm{~g}}{\mathrm{Xbo}}$

After designing the controller it is tested using simulation for different conditions. 
In last section the performance of 1-DOF controller is discussed. The next step is to validate the performance of 2-DOF controller. In order to do a comparative analysis both the controllers are tested under same conditions with same values of PID gains. In this section the significance of each parameter in 2-DOF structure is also presented.

The Maglev system response 2-DOF PID controller with external disturbance is Fig 7

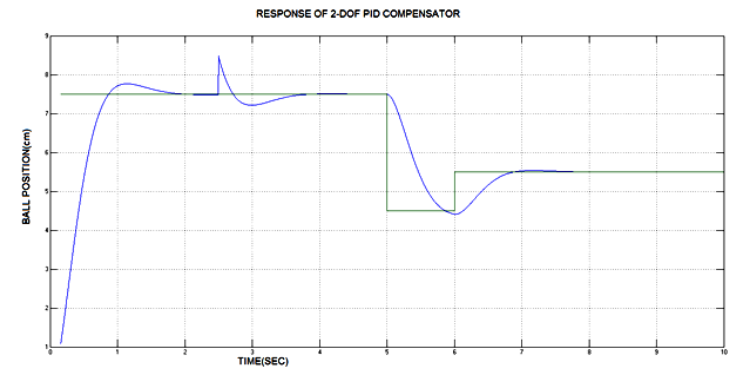

Fig.7 2-DOF PID compensator system response

It can be inferred from the above result that the 2DOF PID controller can handle both setpoint changes and external disturbances at in a very efficient manner. The controller helps the system to follow the trajectory even after the failure.

a. Significance of q2:

Both the feed forward components play a major role in the system response. They help the controller in disturbance rejection by providing addition integral action. q2 plays an important role in the case periodic disturbance. The Fig.8 shows the Maglev system response with periodic disturbance when $\mathrm{q} 2=0$

The periodic disturbance is applied by adding a sinusoidal signal as disturbance. From Fig. 8 it can be inferred that the system is unable to track the periodic disturbance without q2. So $\mathrm{q} 2$ plays an important role in the case of periodic disturbance. The periodic disturbance with $\mathrm{q} 2=\mathrm{q} 1 / 2$ is shown in Fig. 9

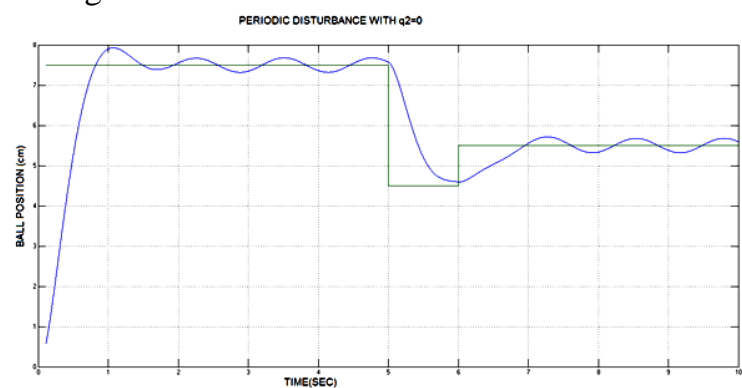

Fig.8 Periodic disturbance with $q 2=0$

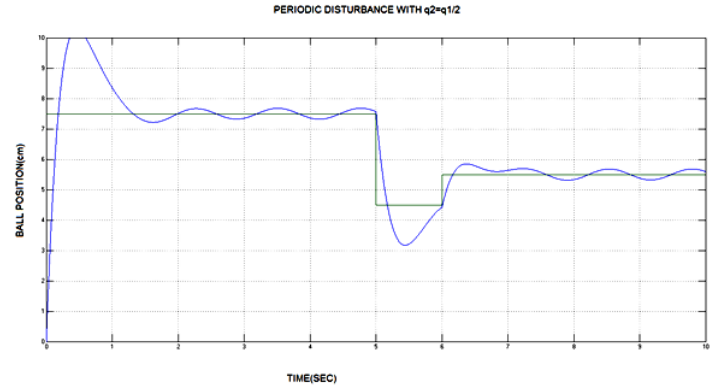


Fig.9 Periodic disturbance with $\mathrm{q} 2=\mathrm{q} 1 / 2$

From the above result it can be inferred that q2 plays a major role in case of periodic disturbance. q2 also offers a trade-off between speed of response and initial oscillations. So q2 acts like a lead compensator. This is evident from the Fig.10

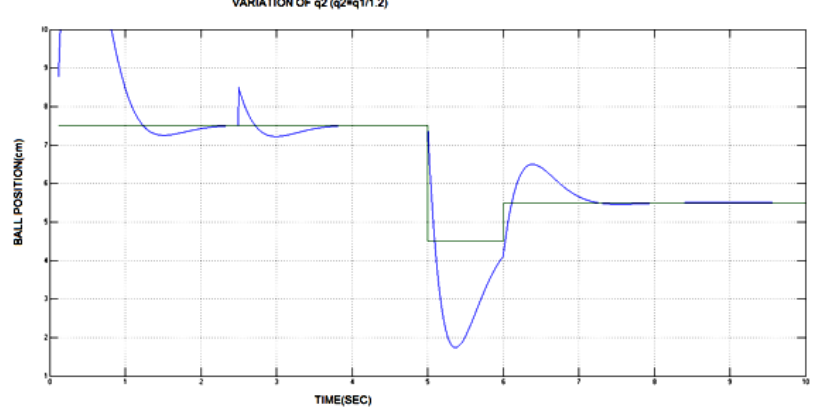

Fig.10 Variation of $q 2(q 2=q 1 / 1.2)$

From the above Fig.10 it is evident that even though there are initial oscillations the speed of response is increased when $\mathrm{q} 2$ is increased.

b. Significance of q1:

q1 acts as an additional integral component. It not only increases the disturbance rejection capability but also improves the steady state response of the system. It acts like a lag compensator. If the value of q1 is decreased there is a steady state error as shown in Fig.11

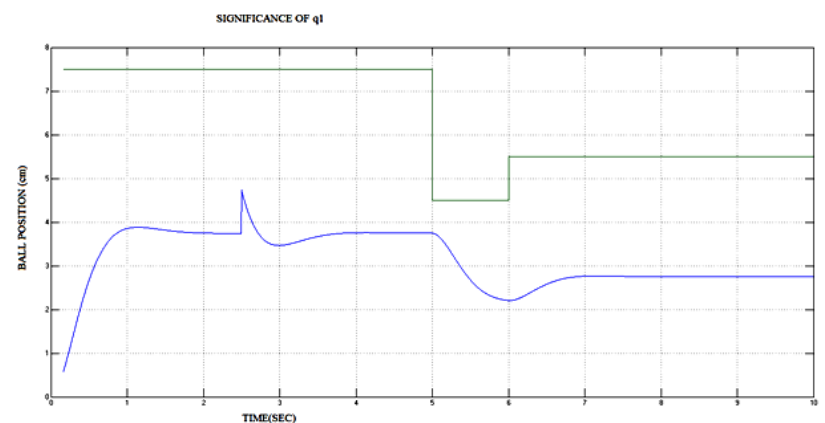

Fig.11 Significance of q1 (q1=Ki/2)

c.Relative stability

As stated above maglev system is an unstable system. So the controllers designed should be able to stabilize the system. The relative stability of the controller helps to find the point at which the controller becomes unstable. It done by introducing a gain block in the output of the controller and it is changed from its nominal value of 1 till the system becomes unstable. The result is as shown in Fig.12

The 2-DOF PID compensator becomes unstable at $\mathrm{k}=0.195$. On comparing the results with the result of 1-DOF controller shown in Fig.5 it is evident that the relative stability of 2DOF PID compensator is efficient over 1DOF PID controller. It makes the system more stable 


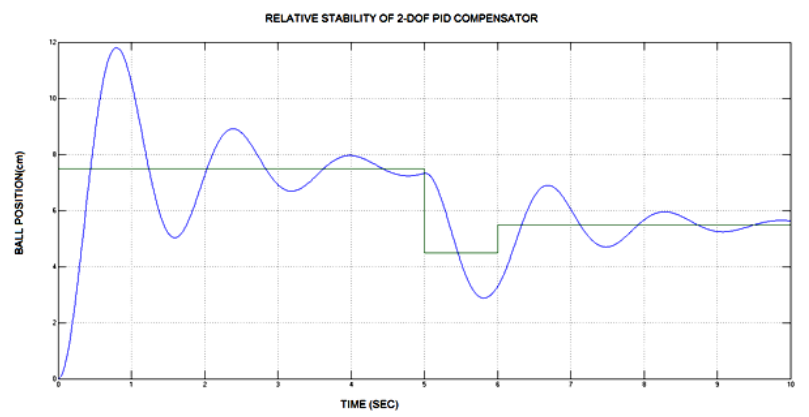

Fig.12 Relative stability of 2-DOF PID compensator

From these results it can be proved that 2-DOF PID compensator is more advantageous than 1-DOF controller. The advantages of 2-DOF compensator for Maglev system are discussed below.

$>$ Due to the presence of additional integral term in the feed forward element the steady state properties are improved.

$>$ The feed forward parameters also help the system to track the set point even after a disturbance is encountered. It helps the system to tackle periodic disturbance also.

$>$ As the Maglev system is an unstable system the controller should stabilize it. When compared with the results of 1-DOF controller 2-DOF controller makes the system stable by decreasing the oscillations and so the loop robustness is improved.

$>$ By using the 2DOF controller, setpoint tracking and interference suppression can be efficiently achieved at the same time.

$>$ The 2-DOF PID compensator serves as a Lead lag compensator for the Maglev system.

\section{Conclusion}

On summarizing the previous chapters it can be concluded that 2-DOF PID controller helps the system to tackle both set point changes and external disturbance simultaneously. It also decreases the over shoot and increases the rise time of the system. It also increases the stability of the system and improves the robustness of the system. So it can be concluded that 2-DOF controller improves the performance of the Maglev system.

The obtained results prove that 2-DOF PID compensator is more advantageous than 1DOF controller. It has shown a great performance when compared with its counterpart in terms of both tracking and regulation. So the next step is to implement it in real time. Hardware implementation will also help in finding many new important conclusions. Now -adays many laboratorykits are available for Maglev system so hardware implementation becomes very easy.

Advances can also be made in the algorithm used. Here 2-DOF controller is used, but Now-a-days 3-DOF controller is under research which can also be applied. In this project the PID gains are found using pole placement technique. There are many advanced methods which can also be applied to find the PID gains some ofthem are Ziegler Nicholas, Cohan coon method many other advanced algorithms like PSO, genetic algorithm can also be used. If these algorithms are applied the system will become more stable. These advances also help in improving the practical importance of the system. 


\section{References}

[1] ArunGhosh , T. Rakesh Krishnan , PaillaTejaswy , AbhisekMandal, Jatin K. Pradhan , SubhakantRanasingh, "Design and implementation of a 2-DOF PID compensation for magnetic levitation systems, , in ISA Transactions, Vol.53(4), pp .1216-1222, 2014.

[2] E. Vinodh Kumar and Jovitha Jerome, "LQR based optimal tuning of PID controller for trajectory tracking of Magnetic Levitation System," Procedia Engineering, Vol.64, pp. 254-264, 2013.

[3] Rafael Morales, Vicente Feliu, and HeberttSira-Ramírez, "Nonlinear Control for Magnetic Levitation Systems Based on Fast Online Algebraic Identification of the Input Gain,” IEEE Trans. Control systems tech, Vol.19(4), pp.757-770, 2011

[4] Faa-Jeng Lin, , Li-Tao Teng, and Po-Huang Shieh, " Intelligent Sliding-Mode Control Using RBFN for Magnetic Levitation System,” IEEE Trans. Industrial Electronics , Vol.54(3), pp.17521762, 2007.

[5] Mei-Yung Chen, Kun-Nan Wu, and Li-Chen Fu," Design, implementation and self-tuning adaptive control of maglev guiding system”, Mechatronics Vol.10 pp.215 - 237,2000.

[6] Zi-Jiang Yang, and MichitakaTateishi ," Adaptive robust nonlinear control of a magnetic levitation system," Automatica 37 pp.1125-1131, 2001

[7] Tania Tariq Salimand Vedat Mehmet Karsli, "Control of Single Axis Magnetic Levitation System Using Fuzzy LogicControl," International Journal of Advanced Computer Science and Applications, Vol. 4(11), pp.84-88, 2013.

[8] Rong-Jong Wai, and Jeng-Dao Lee, " Adaptive Fuzzy-Neural-Network Control for Maglev Transportation System,” IEEE Trans. Neural Networks Vol.19(1), pp.54-69, 2008.

[9] Chin-Teng Lin,and Chong-Ping Jou , "GA-Based Fuzzy Reinforcement Learning for Control of a Magnetic Bearing System”, IEEE Trans. Cybernatics, Vol. 30(2), pp.276-289,2000

[10] MrunalDeshpande, "Optimization of Magnetic Levitation System", International Journal of Engineering Research and Development, Vol.8(7), pp.69-74,2013.

[11] Mituhiko Araki and Hidefumi Taguchi, "Two-Degree-of-Freedom PID Controllers," International Journal of Control, Automation and Systems, Vol. 1, No. 4, pp. 401-412, 2003.

[12] Tan kk, Lee TH and Wang QG, "Enhanced automatic tuning procedure for process control of PI/PID controllers," Procedia Vol.42, pp.2555-62,1999.

[13] Wang Q-G, Fung H-W and Zhang Y, "PID tuning with exact gain and phase margins," ISA Trans. Vol.38 pp.243-49, 1999. 